\title{
MutaScope: a high-sensitivity variant caller dedicated to high-throughput PCR amplicons sequencing
}

\author{
Shawn Yost ${ }^{1,2^{*}}$, Hakan Alakus ${ }^{2,3,4}$, Hiroko Matsui ${ }^{2,3}$, Kristen Jepsen ${ }^{2,3}$, Richard Schwab ${ }^{3,5}$, Kelly Frazer ${ }^{2,3,5,6}$, \\ Olivier Harismendy ${ }^{2,3,5}$
}

From Beyond the Genome 2012

Boston, MA, USA. 27-29 September 2012

With the progress of genomics and targeted therapies, an increasing number of cancer somatic mutations are becoming clinically actionable: predictive of drug sensitivity or resistance. However, clinical samples are often suboptimal for their comprehensive detection. Indeed, contamination with normal cells or the presence of diverse subclones affects their detection by high-throughput sequencing. Ultra-deep targeted sequencing (UDTSeq) is an assay combining microdroplet PCR amplification of exonic sequences followed by a direct, oriented sequencing at high depth of coverage, therefore allowing the detection of low prevalence mutations [1]. Standard sequencing analysis tools that were developed to analyze whole genome and exome shotgun sequencing do not take advantage of UDT-Seq's specific design where each sequencing read originates from a known strand and location. We propose a complete analysis package (MutaScope) dedicated to the analysis of UDT-Seq or similar PCR-based high-throughput sequencing. After alignment to the genome, MutaScope separates the sequencing reads with respect to the amplicons and strand of origin. This allows the experimental measurement of an error rate along the amplicons, which is used to calculate a variant likelihood and rank candidate mutations. Using a set of reference samples, or matched normal DNA, MutaScope then identifies germline and somatic variants and reports them in a unified expanded variant call format. The performance of MutaScope was evaluated on 676 amplicons using a set of calibration samples harboring variants at defined prevalence down to $1 \%$. Overall, MutaScope's sensitivity and positive predictive value
(PPV) were $>96 \%$ and $>75 \%$, respectively; which is higher than the standard variant calling strategies (approximately $70 \%$ and $70 \%$, respectively). MutaScope detects more than $73 \%$ of the variants with an alternate allele frequency $\leq 5 \%$, while the other methods only detect $30 \%$ of the variants. MutaScope offers an analysis strategy specifically dedicated to the identification of low prevalence somatic mutations in high-throughput direct sequencing of PCR amplicons. As a result MutaScope increases the overall technical performance of such approaches that are currently being implemented in clinical diagnostics laboratories.

\section{Author details}

Bioinformatics and Systems Biology Graduate program, University of California, San Diego, La Jolla, CA, USA. ²Division of Genome information Sciences, Department of Pediatrics and Rady Children's Hospital, University of California, San Diego, La Jolla, CA, USA. ${ }^{3}$ Moores UCSD Cancer Center, La Jolla, CA, USA. ${ }^{4}$ Department of General, Visceral and Cancer Surgery, Center for Integrated Oncology, University of Cologne, Germany. ${ }^{5}$ Clinical and Translational Research Institute, University of California San Diego, La Jolla, CA, USA. Institute for Genomic Medicine, University of California San Diego, La Jolla, CA, USA.

Published: 1 October 2012

\section{Reference}

1. Harismendy O, Schwab RB, Bao L, Olson J, Rozenzhak S, Kotsopoulos SK, Pond S, Crain B, Chee MS, Messer K, Link DR, Frazer KA: Detection of low prevalence somatic mutations in solid tumors with ultra-deep targeted sequencing. Genome Biol 2011, 12:R124.

doi:10.1186/1753-6561-6-S6-022

Cite this article as: Yost et al: MutaScope: a high-sensitivity variant caller dedicated to high-throughput PCR amplicons sequencing. BMC Proceedings 2012 6(Suppl 6):O22.

'Bioinformatics and Systems Biology Graduate program, University of

California, San Diego, La Jolla, CA, USA

Full list of author information is available at the end of the article

( 2012 Yost et al; licensee BioMed Central Ltd. This is an Open Access article distributed under the terms of the Creative Commons Attribution License (http://creativecommons.org/licenses/by/2.0), which permits unrestricted use, distribution, and reproduction in any medium, provided the original work is properly cited. 